\title{
PG 1657+416: a new fast pulsating sdB star
}

\section{Photometric observations and theoretical model comparison}

\author{
R. Oreiro ${ }^{1,2}$, F. Pérez Hernández ${ }^{1,2}$, R. Østensen ${ }^{3}$, J. E. Solheim ${ }^{4}$, J. MacDonald ${ }^{5}$, and A. Ulla ${ }^{6}$ \\ 1 Instituto de Astrofísica de Canarias, 38200 La Laguna, Spain \\ e-mail: ror@iac.es \\ 2 Dpto. de Astrofísica, Universidad de La Laguna, 38200 Tenerife, Spain \\ Isaac Newton Group, Aptdo. correos 321, 38700 Santa Cruz de la Palma, Spain \\ ${ }^{4}$ Institute of Theoretical Astrophysics, University of Oslo, PO Box 1029, Oslo, Norway \\ 5 Department of Physics and Astronomy, University of Delaware, DE 19716, USA \\ ${ }^{6}$ Dpto. Física Aplicada, Universidade Vigo, 36310 Vigo, Spain
}

Received 1 March 2006 / Accepted 15 August 2006

\section{ABSTRACT}

\begin{abstract}
Context. Since the discovery in 1997 of stellar oscillations in some B-type hot subdwarfs (sdBs), much effort is being made both observationally and theoretically to characterize this new group of pulsating objects.

Aims. To increase the number of members of such pulsators and to perform a theoretical analysis of new pulsating stars.

Methods. The Nordic Optical Telescope was used to measure the brightness as function of time for a list of selected sdBs. Pulsator candidates were selected by deriving the effective temperatures and gravities of sdB stars from various spectroscopic surveys, and picking targets in the instability region. Pulsational properties were investigated by Fourier analysis. For the theoretical analysis, the predicted frequencies of sdB structural models in the $T_{\text {eff }}-\log g$ region of interest were computed.

Results. From our list of candidates we detected one, PG 1657+416, as a new oscillating star. Follow-up photometry revealed at least four frequencies in the range $6.9-7.9 \mathrm{mHz}$, whose amplitudes vary from one data set to another. The theoretical comparison indicates that the observed periods are well reproduced by structural models whose physical parameters are in good agreement with the effective temperature and gravity estimates from a spectral energy distribution fit, which also shows that the sdB has a G5 main sequence companion with $T_{\text {eff }} \sim 5500 \mathrm{~K}$.
\end{abstract}

Key words. stars: early-type - stars: subdwarfs - stars: oscillations - stars: individual: PG 1657+416

\section{Introduction}

We report the discovery of another member of the class of rapidly pulsating B-type hot subdwarfs (sdBs) named V361 Hya, or EC 14026 stars, after the prototype.

SdBs are believed to be the field counterparts of the Extreme Horizontal Branch stars in globular clusters and have a canonical mass of $0.5 M_{\odot}$, even though there is still some debate concerning their mass distribution and their prior evolution, especially including binary formation (Han et al. 2002, 2003).

In 1997, pulsations in sdBs were predicted (Charpinet et al. 1996), and detected observationally (Kilkenny et al. 1997). The presence of stellar oscillations in sdBs opened up the possibility of employing asteroseismological techniques for this group of evolved objects (Charpinet et al. 2005a,b; Oreiro et al. 2005; Silvotti et al. 2002; Brassard et al. 2001). V361 Hya stars have pulsations with frequencies in the range $2-10 \mathrm{mHz}$ that are explained by p-modes, both radial and non radial, of low degree and low order. The $\kappa$ mechanism associated with an opacity bump due to an enhanced iron abundance in their envelopes, the result of the interplay between gravitational settling and radiative levitation, is responsible for the excitation of stellar oscillations in these stars (Charpinet et al. 1997). The $T_{\text {eff }}$ and $\log g$ of V361 Hya stars, between $28400-35700 \mathrm{~K}$ and 5.25-6.11 dex, place them in an area of the HR diagram where they coexist with non-variable sdBs. A possible explanation for this co-existence comes from mass loss, which is thought to play an important role in emptying the iron reserve (Chayer et al. 2004).

\section{Observations}

The star PG $1657+416$ was selected from a catalogue of identified hot subdwarfs (Østensen 2004), whose source in this particular case was the SDSS First Data Release. The target's high infrared excess (see Table 1) clearly identifies it as a hot subdwarf plus main sequence binary system (see eg. Stark \& Wade 2003).

PG 1657+416 was included in our sample only after preliminary temperature and gravity estimates were obtained from fits to Sloan archive spectroscopy. Although the presence of a companion makes a direct spectroscopic fit unreliable, our first estimate of its $T_{\text {eff }}$ is $33900 \mathrm{~K}$, which would place the star within the hot end of the instability region of the rapidly pulsating EC 14026 stars. We used the Nordic Optical Telescope (NOT) located on La Palma (Canary Islands, Spain) to observe the object during a three-night campaign devoted to search for such oscillators.

As it was observed during the last night of the campaign (May 25th, 2004, see Table 2 for details), and almost at sunrise, its pulsating behaviour was only marginally detected, as shown in the upper panel of Fig. 1. For this reason the object was re-observed in a subsequent photometric campaign in 
Table 1. Photometric information available on the target.

\begin{tabular}{cccccccc}
\hline \hline \multicolumn{2}{l}{ SDSS name: J165841.83+413115.6 } & \multicolumn{4}{c}{ Alias: PG 1657+416 } \\
\hline$u$ & $g$ & $r$ & $i$ & $z$ & $J$ & $H$ & $K_{\mathrm{S}}$ \\
\hline 15.81 & 15.94 & 16.13 & 16.27 & 16.35 & 15.80 & 15.64 & 15.13 \\
\hline
\end{tabular}

Table 2. Date, UT of the first exposure of each run, its corresponding length, telescope and observers for all the photometric campaigns: discovery, confirmation, IAC80 and NOT2005.

\begin{tabular}{ccccc}
\hline \hline Date & UT start & Length & Telescope & Observer \\
\hline May, 25, 2004 & $4: 40: 00$ & $32 \mathrm{~m}$ & NOT & RØ/RO \\
\hline June, 6, 2004 & $3: 46: 53$ & $55 \mathrm{~m}$ & NOT & JES \\
\hline May, 4, 2005 & $22: 51: 20$ & $6 \mathrm{~h} 33 \mathrm{~m}$ & & \\
May, 5, 2005 & $22: 40: 03$ & $6 \mathrm{~h} 38 \mathrm{~m}$ & & \\
May, 6, 2005 & $23: 19: 15$ & $5 \mathrm{~h} 58 \mathrm{~m}$ & IAC80 & RO \\
May, 7, 2005 & $23: 41: 18$ & $2 \mathrm{~h} 23 \mathrm{~m}$ & & \\
May, 8, 2005 & $23: 25: 13$ & $5 \mathrm{~h} 41 \mathrm{~m}$ & & \\
\hline May, 30, 2005 & $23: 18: 11$ & $1 \mathrm{~h} 26 \mathrm{~m}$ & & \multirow{2}{*}{ RO } \\
May, 31, 2005 & $22: 55: 18$ & $2 \mathrm{~h} 0 \mathrm{~m}$ & NOT & \\
June, 1, 2005 & $23: 42: 30$ & $1 \mathrm{~h} 28 \mathrm{~m}$ & & \\
\hline
\end{tabular}

which the presence of periodic modulations in its light curve was confirmed.

The same telescope (NOT) and instrument (ALFOSC in fast photometry mode) were used for the second campaign. PG 1657+416 was observed for 55 min on June 6th, 2004 (see Table 2 for details), and the amplitude spectrum displayed in the lower panel of Fig. 1 was obtained. Some signal can be easily seen in the region of $7-8 \mathrm{mHz}$, thus confirming the oscillatory nature of the object. The noise levels shown in the figures are computed as the mean amplitude of the spectra before prewhitening.

The frequency analysis of the discovery and confirmation runs leads to the frequencies listed in Table 3. Bearing in mind their low resolution (as indicated by the $F W H M$ in the table), two peaks $\left(f_{1}=6.8, f_{2}=7.8 \mathrm{mHz}\right)$ with equal amplitude $\left(2.5 \mathrm{mma}^{1}\right)$ were suggested in the discovery run, while during the confirmation run (see also Table 3 ), $f_{1}$ had a lower amplitude (1.8 mma) while the dominant peak, with amplitude $3.7 \mathrm{mma}$, was at $f_{3}=$ $7.5 \mathrm{mHz}$.

In spite of the relative faintness of the $\operatorname{star}(B=15.87$, Green et al. 1986), we used one week of observing time at the $80 \mathrm{~cm}$ IAC80 telescope located on Tenerife. The use of no filter, together with a high cycle time (40 s) and a fast readout obtained by observing in window mode, which allowed exposure times as long as $37 \mathrm{~s}$, were needed in order to optimize the quality of the data obtained with a telescope of this limited size. Information about the observations is given in Table 2. The data were reduced with the Real Time Photometry (RTP) software (Østensen 2000): bias correction, sky subtraction, division by a comparison star and extinction correction were applied. The useful data after this process gave the light curve shown in the upper panel of Fig. 2.

At the top of Fig. 3 the spectral window that corresponds to the time series of the IAC 80 data is shown. It displays a $F W H M$ of $3 \mu \mathrm{Hz}$, which is adopted as the frequency resolution. A closer view of the spectral window is enclosed in the top-right corner, although within the main panel the same frequency scale as that used in the panels below is employed. From the second to the last panel of Fig. 3 the amplitude spectrum and the

\footnotetext{
1 mma corresponds to millimodulation amplitude, with $1 \mathrm{mmag} \simeq$ $1.08 \mathrm{mma}$.
}
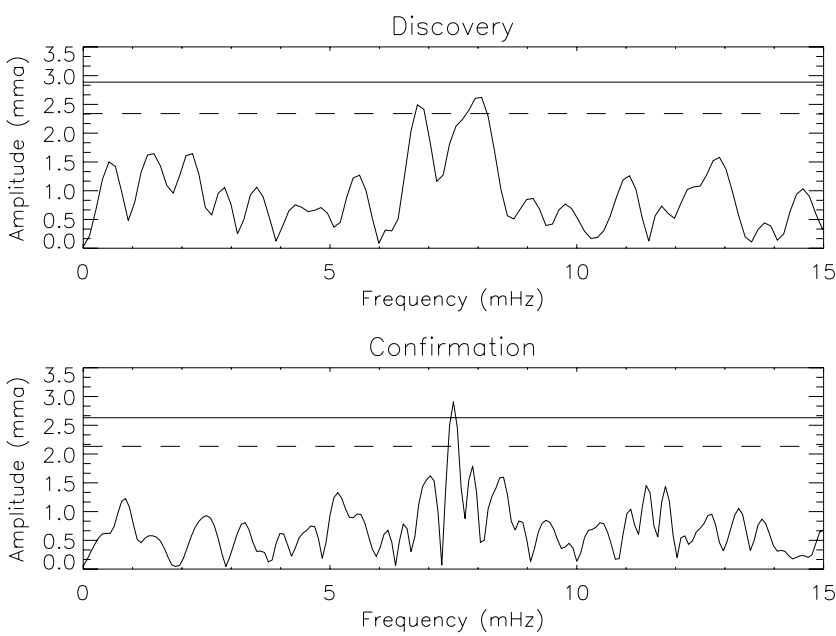

Fig. 1. Amplitude spectrum of PG $1657+416$ obtained with the NOT in May, 2004 (discovery run, upper panel) and that obtained with the NOT in June, 2004 (confirmation run, lower panel). The continuous horizontal lines indicate 3.7 times the noise level (in amplitude), the dashed lines indicate 3 times the noise level.

Table 3. Frequency, period, amplitude, phase and signal-to-noise ratio of the pulsations detected in the amplitude spectrum of PG 1657+416 in the different runs. Phase is relative to the first data point of each light curve. The $\mathrm{S} / \mathrm{N}$ values are after prewhitening.

\begin{tabular}{cccccccc}
\hline \hline Run & $\begin{array}{c}F W H M \\
(\mu \mathrm{Hz})\end{array}$ & \multicolumn{2}{c}{ Freq. } & Period Ampl. & Phase & $S / N$ \\
& $(\mu \mathrm{Hz})$ & $(\mathrm{s})$ & $(\mathrm{mma})$ & $(\mathrm{rad})$ & \\
\hline Discovery & 520 & $f_{1}$ & 6857 & 145 & 2.52 & 2.11857 & 4.37 \\
& & $f_{2}$ & 7880 & 126 & 2.52 & 3.05380 & 3.08 \\
\hline Confirmation & 303 & $f_{1}$ & 6969 & 143 & 1.78 & -0.16974 & 4.66 \\
& & $f_{3}$ & 7500 & 133 & 3.71 & 1.92694 & 4.62 \\
\hline IAC80 2005 & 3 & $f_{1}$ & 6891.94 & 145 & 1.61 & 0.84404 & 4.61 \\
& & $f_{4}$ & 7731.37 & 129 & 1.36 & -3.40146 & 3.52 \\
& & $f_{5}$ & 7150.18 & 139 & 1.23 & -0.13925 & 3.68 \\
& & $f_{3}$ & 7568.54 & 132 & 1.17 & -0.51874 & 3.16 \\
\hline NOT 2005 & 4.8 & $f_{3}$ & 7567.74 & 132 & 2.69 & 0.74745 & 15.15 \\
& & $f_{4}$ & 7730.67 & 129 & 0.96 & 0.23507 & 5.97 \\
& & $f_{2}$ & 7831.95 & 139 & 1.17 & 1.76060 & 6.95 \\
\hline IAC80+NOT & 2.95 & $f_{1}$ & 6891.88 & 145 & 1.34 & 0.90497 & 4.51 \\
2005 & & $f_{3}$ & 7568.66 & 132 & 1.44 & -0.67775 & 4.65 \\
& & $f_{4}$ & 7731.21 & 129 & 1.28 & 3.06778 & 3.95 \\
& & $f_{5}$ & 7150.10 & 139 & 1.01 & -0.04592 & 3.63 \\
\hline
\end{tabular}

subsequent spectra of its prewhitening process are shown. The continuous horizontal lines indicate 3.7 times the noise level, while the dashed horizontal ones mark 3 times this value. The vertical dashed lines correspond to the frequencies that are successively subtracted from the signal. The results of a non-linear least squares fit are included in Table 3, where amplitudes, phases and signal-to-noise ratio are given for four frequencies.

Comparing the amplitudes in the spectrum from the NOT (Fig. 1) and that from the IAC80 (Fig. 3) some differences are evident. The latter shows a much lower amplitude, suggesting that beating effects may be occurring. The light curve from the IAC80 recovers $f_{1}$ as the dominant peak, followed in amplitude by $f_{4}=7.7 \mathrm{mHz}$, while $f_{3}$ (dominant in the confirmation run) is only 3.16 times above the noise level. Also, a new frequency $\left(f_{5}=7.1 \mathrm{mHz}\right)$ with a slightly higher signal-to-noise ratio (3.68) is found. A marginal peak with a similar signal to noise ratio as that of $f_{3}$ is still seen at $v=7.798 \mathrm{mHz}$ in the residual amplitude spectrum. Contrary to $f_{3}$, however, this marginal peak was 

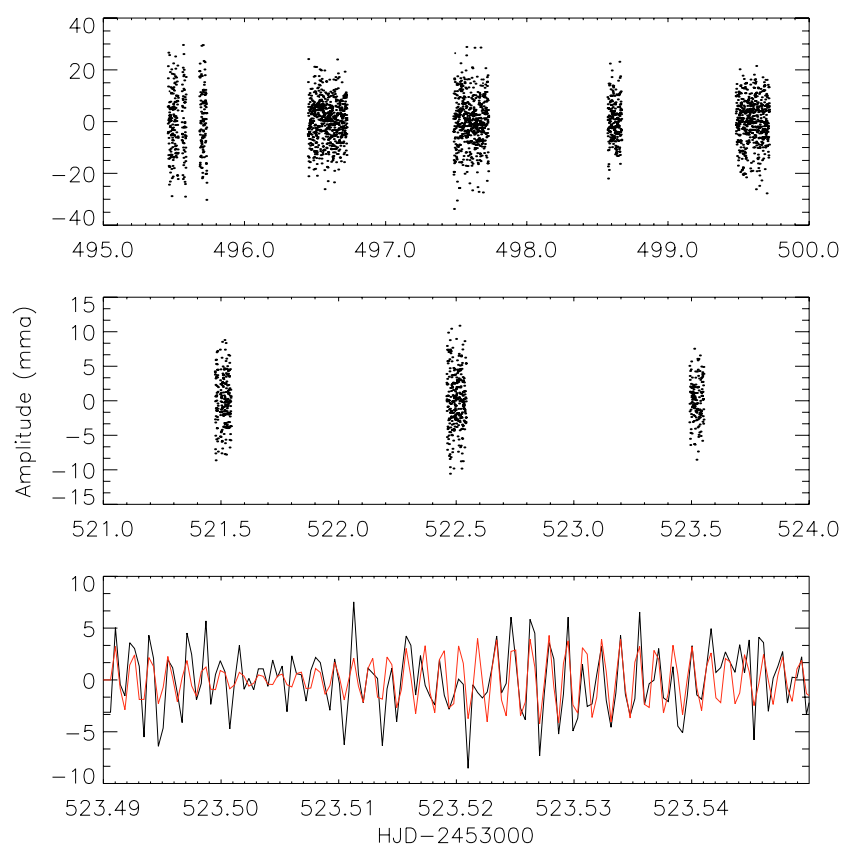

Fig. 2. Upper: light curve of PG $1657+416$ obtained with the IAC 80 telescope in May, 2005; Middle: light curve obtained with the NOT in May, 2005; Bottom: detail of the light curve obtained with the NOT and the fit to a synthetic light curve (in red), computed from the frequencies derived from these data, and listed in Table 3.

not recovered in the subsequent photometric campaign, detailed below, and thus this frequency is not considered further.

Three weeks later, about one and a half hours on each of three nights were employed to observe PG $1657+416$, see Table 2 for details. Reduced in the same way as previously, the derived light curve is shown in the middle panel of Fig. 2. The bottom panel displays a detail of this light curve, where a synthetic light curve has been overplotted as a red line. The synthetic light curve was computed using the frequencies derived from the frequency analysis, which are included in Table 3.

The light curve of the star obtained during the run NOT-2005 resulted in the amplitude spectrum shown in Fig. 4, whose spectral window (shown in the upper panel) displays a $F W H M=$ $4.8 \mu \mathrm{Hz}$. The frequency analysis now leads to $f_{3}$ having the highest amplitude (as in the confirmation run), and the signal around $7.8 \mathrm{mHz}$ splits into two peaks $\left(f_{2}, f_{4}\right)$ with amplitudes both above 3.7 times the noise level. However, $f_{1}$ is not detected in these data. The frequency list from the run NOT-2005 is given in Table 3.

Although 22 nights separate the last run taken at the IAC80 from the first at the NOT, we attempted to combine the data to improve the frequency resolution and also to avoid possible mistakes coming from the high side-bands of the spectral window obtained at the NOT. Figure 5 summarizes the results from the analysis, showing the spectral window, the amplitude spectrum and the prewhitening process. The derived frequencies are listed in Table 3.

The amplitudes of the detected peaks differ from one data set to another, from no detection to being the dominant peak. This would imply that either the detected frequencies suffer from beating effects due to unresolved peaks, or that the detected frequencies undergo intrinsic amplitude variations. The data presented here do not allow a deeper analysis in terms of signal to noise ratio or time coverage to distinguish between the two options. However, all the detected frequencies should have an

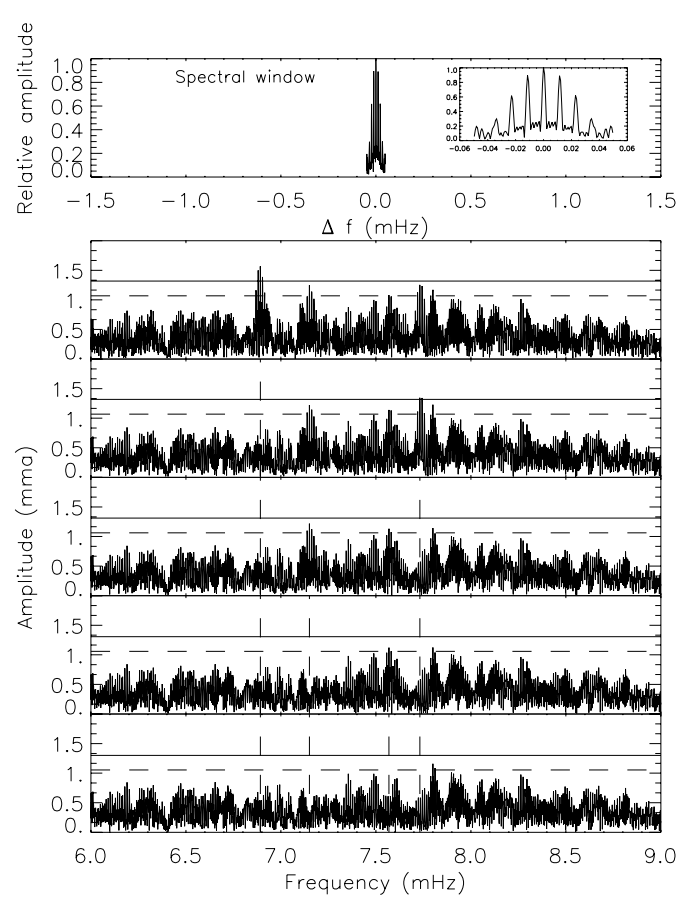

Fig. 3. Run: IAC80 May, 2005. From top to bottom: spectral window, amplitude spectrum, and those after successively subtracting peaks from the signal. The continuous horizontal line indicates 3.7 times the noise level, whereas the dashed one corresponds to 3 times this level. The vertical dashed lines mark the frequencies that are removed during the prewhitening process.
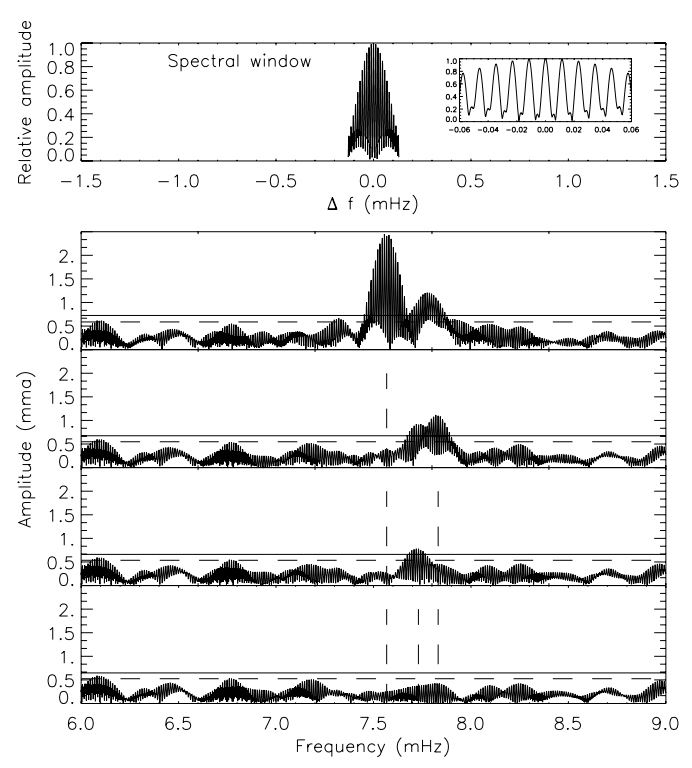

Fig. 4. Run: NOT May, 2005. From top to bottom: spectral window, amplitude spectrum, and those after successively subtracting peaks from the signal. The continuous horizontal line indicates 3.7 times the noise level, whereas the dashed one corresponds to 3 times this level. The vertical dashed lines mark the frequencies that are removed during the prewhitening process.

unresolved close frequency if we favour the beating solution. On the other hand, the suggestion of amplitude variations is not new for pulsating sdB stars, as this is the case for PG 1219+534 (Harms et al. 2005) or HS 1824+5745 (Reed et al. 2006), for example. Further photometric campaigns are needed to better understand the amplitude spectrum of PG $1657+416$. 


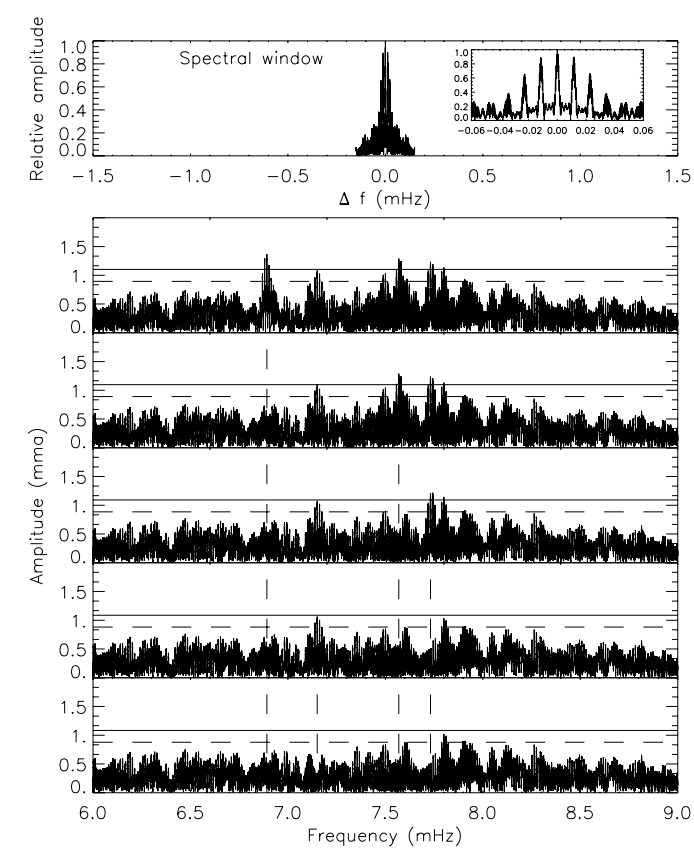

Fig. 5. Run: IAC80+NOT 2005. From top to bottom: spectral window, amplitude spectrum, and those after successively subtracting peaks from the signal. The continuous horizontal line indicates 3.7 times the noise level, the dashed one corresponds to 3 times the noise. The vertical dashed lines mark the frequencies that are removed during the prewhitening process.

Despite the amplitude variations, the frequencies $f_{2}, f_{3}$ and $f_{4}$ are above 3.7 times the noise level in the NOT-2005 run. Besides being the dominant peak in the discovery run, $f_{1}$ is above 3.7 times the noise level in the confirmatory observations, and is again the frequency with the highest amplitude in the IAC80 campaign. On the other hand, $f_{5}$ is only detected in the latter campaign, in which its significance is close to the confidence limit. For these reasons, we will only consider $f_{1}, f_{2}, f_{3}$ and $f_{4}$ for the theoretical analysis in next section.

\section{Theoretical comparison}

\section{1. $\log g$ determination}

Knowing the $\log g$ and $T_{\text {eff }}$ of the target is desirable to compare the observed and theoretical frequencies. However, as mentioned before, PG $1657+416$ has a companion star that influences both the photometric magnitudes and the observed spectrum of the star. For this reason, an accurate fit to the Balmer lines is not easily achieved, although a first estimate of the sdB effective temperature gives $T_{\text {eff }} \sim 33900 \mathrm{~K}$.

An alternative method to compute the sdB parameters is that described in Koen et al. (1997), in which the observed flux of the composite system, coming from spectroscopy or from photometry, is fitted to a sum of two spectra following the relation:

$F_{v}=\left(\frac{r_{1}}{d}\right)^{2} S_{1}\left(T_{1}, v\right)+\left(\frac{r_{2}}{d}\right)^{2} S_{2}\left(T_{2}, v\right)$

where $d$ is the distance to the system, and $r_{i}, T_{i}$ and $S_{i}$ are the radius, temperature and flux emission of each object.

Prior to any fit, both the infrared magnitudes, converted to fluxes using the factors in Heber et al. (2002), and the flux from the spectrum were corrected for reddening effects following:

$F_{\lambda}^{0}=F_{\lambda} \times 10^{A_{V} f^{\prime}(\lambda) / 2.145}$

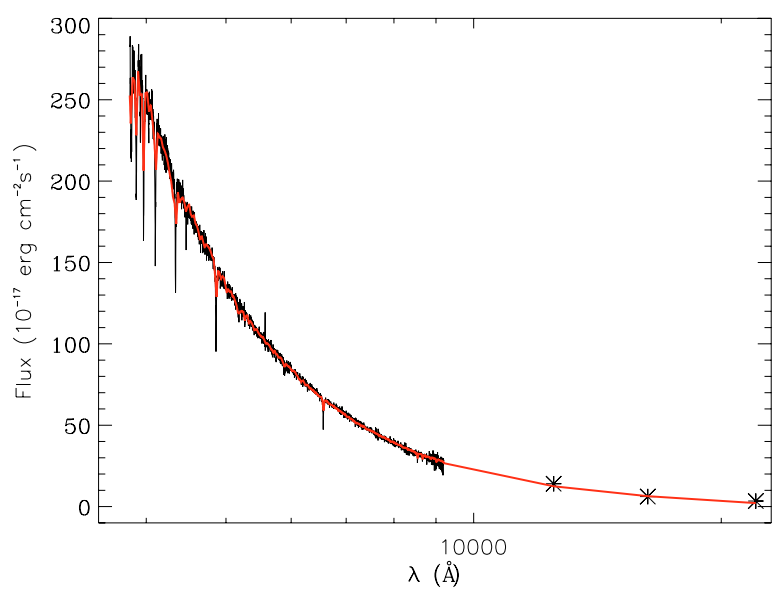

Fig. 6. Observed spectrum, 2MASS magnitudes converted to fluxes (asterisks) and the best fit to a sum of two Kurucz model atmospheres obtained by adding a spectrum with $T_{\text {eff }}=5500$ and $\log g=4$ to a sdB model with $T_{\text {eff }}=34000$ and $\log g=5$.

where $F_{\lambda}^{0}$ and $F_{\lambda}$ are the corrected and uncorrected fluxes, $A_{V}=$ $3.1 E(B-V)$, the color excess obtained from the maps of Schlegel et al. (1998), and $f^{\prime}(\lambda)$ taken from Whitford (1958), which can be approximated in the whole spectral range with an accuracy better than $5 \%$ by $f^{\prime}(\lambda)=3.15854 \times 10^{-1.02109 \lambda}$.

A least squares fit was performed to the corrected observed fluxes (spectrum from SDSS with a $S / N \sim 40$ and magnitudes from 2MASS). As a first step, Kurucz model atmospheres were used as $S_{i}$ functions, keeping the sdB temperature fixed to the line fitting estimate $\left(T_{\text {eff }} \sim 34000 \mathrm{~K}\right)$ and varying the companion temperature from 3500 to $7500 \mathrm{~K}$, in steps of $250 \mathrm{~K}$. Model atmospheres with $\log g=4$ and 4.5 were used for the red star, while a model with $\log g=5$ was employed to represent the hot subdwarf.

The best fit to the continuum, in the sense of a minimum $\chi$-square error, is obtained when an atmosphere with $T_{\text {eff }}$ between 5250 and $5750 \mathrm{~K}$ is used for the companion. In Fig. 6 the observed spectrum and magnitudes, together with the best fit for the $\left(T_{\text {eff }}=5500, \log g=4\right),\left(T_{\text {eff }}=34000, \log g=5\right)$ combination is shown.

If the red star is assumed to be on the main sequence, then its effective temperature gives an estimate of its radius. According to main sequence structural models from the ZAMS to the near end of the H-core burning (Claret 1995), a star with $T_{\text {eff }}$ in the range $5250-5750 \mathrm{~K}$ has a radius in the range $0.822-1.4 R_{\odot}$. As the coefficients of the flux fit give the $r_{1} / r_{2}$ ratio, the sdB radius is thus expected to be in the range $0.124-0.242 R_{\odot}$ and, by assuming that the sdB mass is $0.47 M_{\odot}$, the resultting $\log g$ for the hot subdwarf lies in the range 5.33-5.92 dex, which are typical values for $\mathrm{sdB}$ stars.

Having obtained a good estimate of the effective temperature for the companion, which we adopt to be $T_{\text {eff }}=5500 \pm 500 \mathrm{~K}$, a new least squares fit to the observed fluxes was made. Higher resolution spectra (Munari et al. 2005) were used in order to have a better treatment of the lines. A $T_{\text {eff }}=5500 \mathrm{~K}, \log g=4$ model and a $T_{\text {eff }}=34000 \mathrm{~K}, \log g=5$ one, with $[\mathrm{Fe} / \mathrm{H}]=-2.5$, were used as $S_{i}$ functions in this case in order to compute the coefficients of Eq. (1), and to enable removal of the companion star contribution to the observed flux.

The last step consists of performing a line profile fit to the residual spectrum, after subtracting the red star contribution to the observed spectrum. The line blanketed LTE spectral synthesis model atmospheres of Heber et al. (2000) were used 


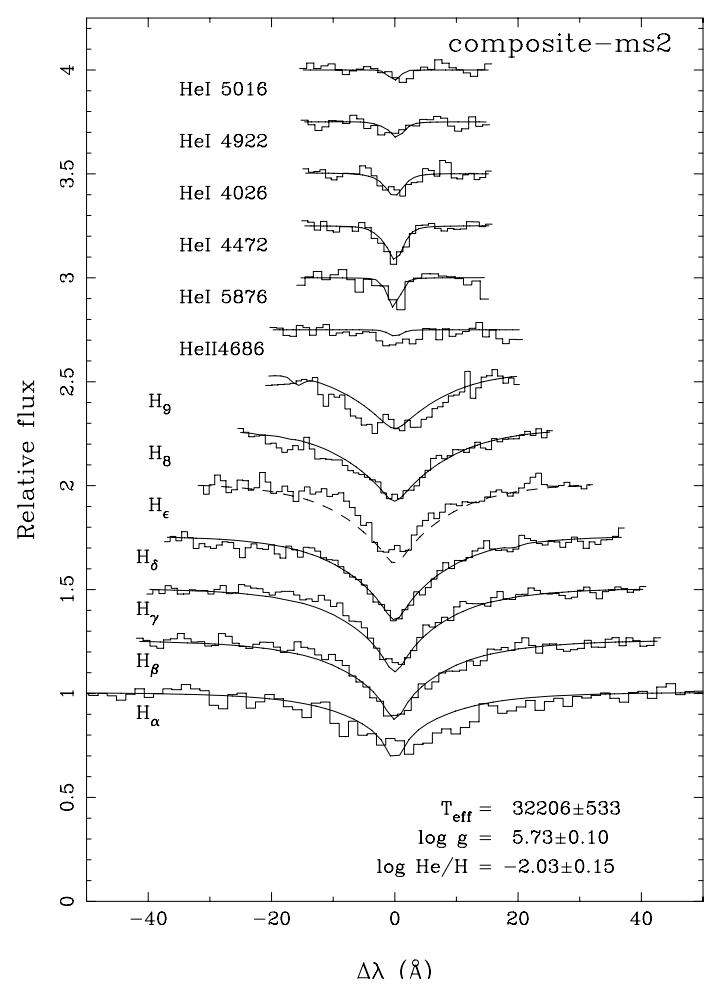

Fig. 7. Best line fit to the observed flux after subtracting a main sequence $T_{\text {eff }}=5500 \mathrm{~K}$ model atmosphere.

in the $\chi^{2}$ procedure of Bergeron et al. (1992) as updated by Saffer et al. (1994). The resulting best fit, shown in Fig. 7, gives $T_{\text {eff }}=32200 \pm 500 \mathrm{~K}, \log g=5.73 \pm 0.10 \mathrm{dex}, \log n(\mathrm{He}) / n(\mathrm{H})=$ $-2.03 \pm 0.15$ for the sdB.

Given the number of assumptions made to calculate the parameters just derived, it is difficult to estimate the relevance of the quoted errors. If for the main sequence star, model atmospheres with $\log g=4.5$ are used instead of those with $\log g=4$, all the parameters remain within the error bars indicated. As these gravities can be considered as extreme cases for a main sequence star with $T_{\mathrm{eff}} \sim 5500 \mathrm{~K}$, we conclude that the choice of an accurate $\log g$ value does not have a significant effect on the results. On the other hand, in the two firsts steps we used $\log g$ values smaller than those typical for sdB stars, but this is not expected to influence the final result, since they are only employed to obtain the relative fluxes according to Eq. (1) (mainly a fit to the continuum).

\subsection{Theoretical frequency comparison}

Given the difficulty in estimating the uncertainties in the stellar parameters derived in the previous section, for the seismic analysis we will make use of sdB structural models in a wider range of $T_{\text {eff }}-\log g$ values.

In Fig. 8 three sdB evolutionary sequences (A, B and C) are plotted, in which ages (in $10^{8}$ years) are given. These tracks were produced by evolving a $1.0 M_{\odot}$ model star from the main sequence through the helium core flash and on to the white dwarf cooling track using the stellar evolution code of Jiménez \& MacDonald (1996). Only part of the complete tracks are shown in Fig. 8. Mass loss on the RGB with Reimer's parameter (Reimers 1975) $\eta=0.8,0.7$ and 0.695 result in tracks A, B and $C$ respectively, whose physical parameters at the beginning of the He-core burning phase are given in Table 4. A dashed error
Table 4. Total mass $(M)$, hydrogen envelope mass $\left(M_{\mathrm{H}}\right), T_{\text {eff }}$ and $\log g$ of the structural models at the beginning of the sdB time evolution of sequences $\mathrm{A}, \mathrm{B}, \mathrm{C}$ and $\mathrm{D}$.

\begin{tabular}{ccccc}
\hline \hline & $M\left(M_{\odot}\right)$ & $M_{\mathrm{H}}\left(M_{\odot}\right)$ & $T_{\text {eff }}(\mathrm{K})$ & $\log g(\mathrm{dex})$ \\
\hline $\mathrm{A}$ & 0.46 & $2.2 \times 10^{-5}$ & 33806 & 6.07 \\
$\mathrm{~B}$ & 0.47 & $2.5 \times 10^{-4}$ & 30620 & 5.87 \\
$\mathrm{C}$ & 0.47 & $3.0 \times 10^{-4}$ & 28675 & 5.72 \\
$\mathrm{D}$ & 0.53 & $2.8 \times 10^{-4}$ & 32395 & 5.80 \\
\hline
\end{tabular}

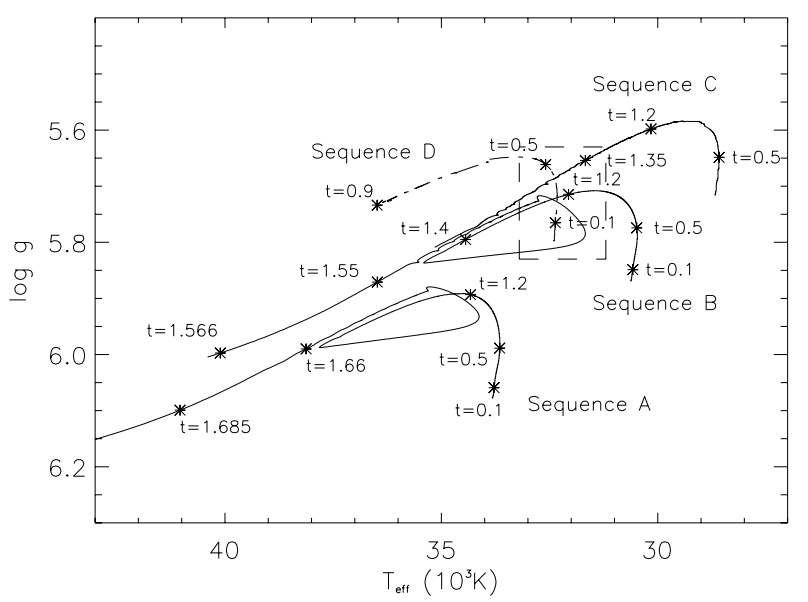

Fig. 8. $\log g-T_{\text {eff }}$ diagram in which three $\mathrm{sdB}$ evolutionary sequences $(\mathrm{A}, \mathrm{B}, \mathrm{C})$ produced by a complete evolution from a main sequence star are displayed. Sequence D was artificially generated so that it has a higher total mass and crosses the star's location at an earlier evolutionary stage. Some ages (in $10^{8}$ years) are given at the points indicated with asterisks. The values of $\log g-T_{\text {eff }}$ obtained in the previous section for PG $1657+416$ are indicated with a dashed error box.

box representing the atmospheric parameters of PG $1657+416$ derived in the previous subsection is also shown in Fig. 8.

The location of a particular sequence in the $\log g-T_{\text {eff }}$ plane depends mainly on the total mass $(M)$ and on the mass of the $\mathrm{H}$ envelope $\left(M_{\mathrm{H}}\right)$. A thinner $\mathrm{H}$ envelope, which could be due to a higher mass loss rate, shifts the whole evolutionary sequence towards higher temperatures and gravities. This is the case for sequences A, B, C, which have almost the same total mass but an increasing envelope mass. A single star formation channel for sdBs of this type produces very similar core masses, independently of the initial conditions because the core mass at helium ignition depends only weakly on initial mass and heavy element abundance, whereas the $\mathrm{H}$ envelope mass can vary depending on the mass loss efficiency during the RGB phase. On the other hand, by increasing the total mass, a sequence with a particular $M_{\mathrm{H}}$ would be shifted towards higher temperatures. To account for this case, an ad hoc sequence (D) was generated with a higher total mass so that it crosses the star's location at an earlier evolutionary stage. Sequence D is indicated in Fig. 8 with a dashed-dotted line.

Although in the rest of the section we assume that the observed peaks correspond to modes of a non-rotating star, a rotational origin cannot completely be rejected. For p-modes the rotational splitting is given by $\Delta v \sim \Delta m v_{\text {rot }}$, where $v_{\text {rot }}$ is the average rotation rate of the star and a difference of $\Delta m \leq 4$ must be considered for modes with $\ell \leq 2$. Choosing a radius of $R \sim 6.5 \times 10^{7} \mathrm{~m}$, corresponding to the minimum of the structural models shown in Fig. 8, the smallest separation between the observed frequencies, $f_{2}-f_{4}=0.101 \mathrm{mHz}$, can be explained as 

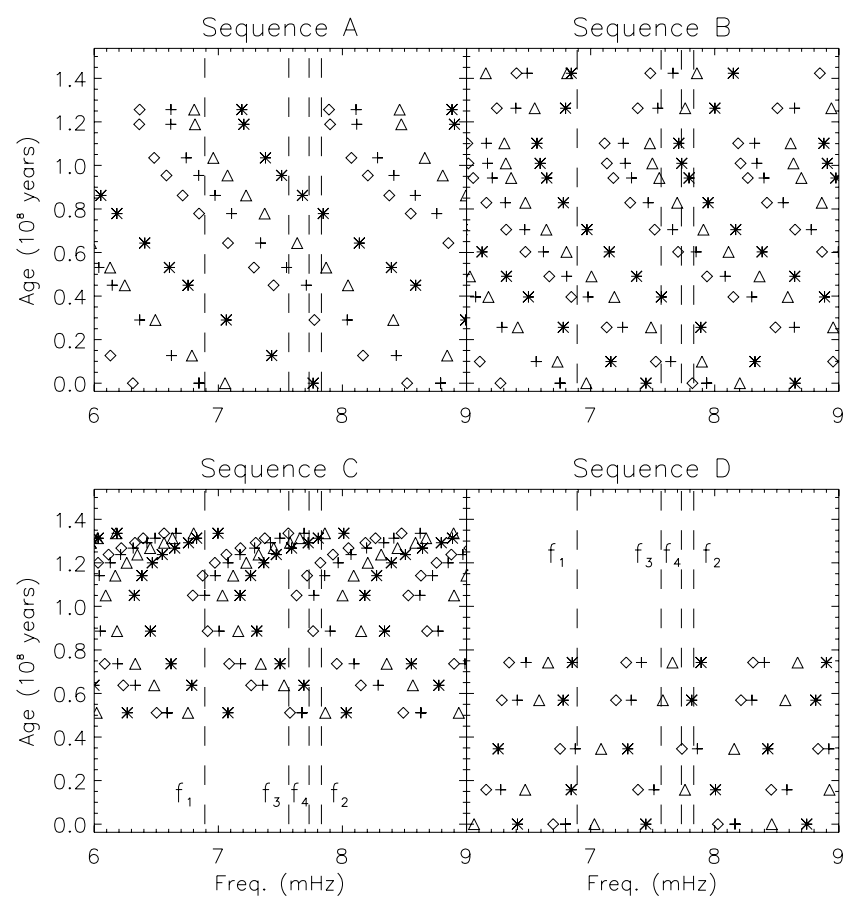

Fig. 9. Theoretical frequencies for structural models of sequences A, B, $\mathrm{C}$ and $\mathrm{D}$. The $y$-axis indicates the age of the models, while the vertical broken lines point out the observed frequencies. Crosses: modes with $\ell=0$; asterisks: models with $\ell=1$; rhombus: $\ell=2$; triangles: $\ell=3$.

a rotational splitting if the star is rotating with a velocity greater than about $10 \mathrm{~km} \mathrm{~s}^{-1}$.

As a first step, we make a crude comparison between the observed frequencies $\left(f_{1}\right.$ to $\left.f_{4}\right)$ and the non-rotating theoretical ones predicted for several structural models belonging to the four sequences. In Fig. 9 we have plotted the theoretical frequencies of those models within a suitable time interval, according to the star's $\log g-T_{\text {eff }}$ values. The $y$-axis indicates the age of the models from the beginning of He-core burning, while the vertical broken lines point out the observed frequencies.

The fundamental mode $(\ell=0, n=1)$ for sequence $\mathrm{A}$ is within $5.36-6.84 \mathrm{mHz}$, while for sequence B the fundamental mode varies from 4.28 to $5.6 \mathrm{mHz}$ and for sequences $\mathrm{C}$ and $\mathrm{D}$ from 3.33-3.85 and 3.79-4.82. The observed frequencies would correspond to modes with radial order $n=0,1,2$ according to models on sequence $\mathrm{A}$, models on sequence $\mathrm{B}$ indicate the frequencies have a radial order $n=2,3$, 4, and models on sequences $\mathrm{C}$ and $\mathrm{D}$ indicate that the frequencies have radial orders $n=4,5,6$ and $n=2,3,4$ respectively.

As can be seen in Fig. 9, matching the observed frequency density formed by $f_{2}, f_{3}$ and $f_{4}$ gives a strong constraint on which model best reproduces the observations. In particular, it appears that models of sequence A do not produce a dense enough spectrum in the frequency range of interest, as can be seen in Fig. 9, while models of sequences B and C show more closely spaced modes.

This fact is better shown in Fig. 10, where the small frequency separation for models along the four sequences are plotted. The frequency separation between $\ell=0$ and $\ell=2\left(\Delta v_{0,2}\right)$ is indicated with crosses, and $\Delta v_{0,3}$ and $\Delta v_{1,3}$ are shown with triangles and asterisks, respectively. The values of $f_{2}-f_{4}=$ $0.101 \mathrm{mHz}$ and $f_{4}-f_{3}=0.163 \mathrm{mHz}$ are overplotted as horizontal broken lines for comparison.

It is easily seen in Fig. 10, as suggested in Fig. 9, that the overall small separation between modes for structural models
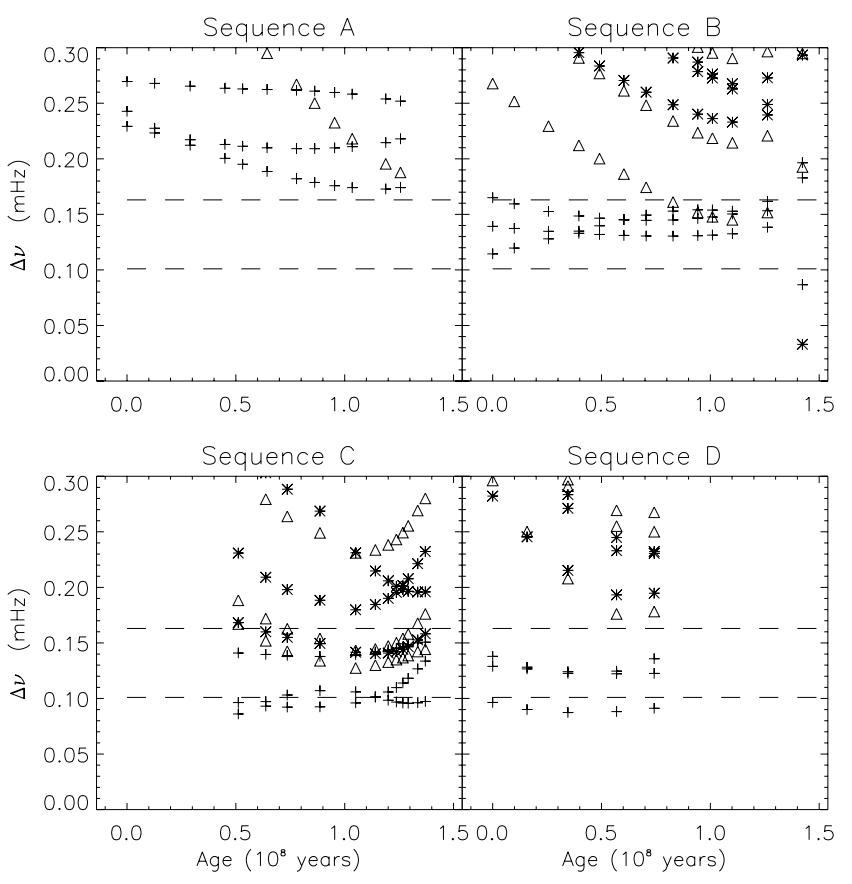

Fig. 10. The frequency separation $\Delta v_{0,2} \equiv v_{\ell=0}-v_{\ell=2}$ (crosses), $\Delta v_{3,0}$ (triangles) and $\Delta v_{1,3}$ (asterisks) for structural models from sequence $\mathrm{A}, \mathrm{B}$, $\mathrm{C}$ and $\mathrm{D}$. The values of $f_{2}-f_{4}=0.101 \mathrm{mHz}$ and $f_{4}-f_{3}=0.163 \mathrm{mHz}$ are overplotted as horizontal broken lines for comparison.

along sequence $\mathrm{A}$ is greater than that for models on other sequences, especially those along sequence C. The observed frequency separations are better reproduced by models of sequences $\mathrm{B}, \mathrm{C}$ or $\mathrm{D}$, which is in agreement with the $T_{\text {eff }}-$ $\log g$ spectroscopic determination.

Besides the separation within the group formed by $f_{2}$ to $f_{4}$, another constraint for theoretical matching is the frequency separation between this group and $f_{1}$. As we are considering four frequencies lying in a close frequency range $(6.9-7.9 \mathrm{mHz})$, two of them must be of the same degree $\ell$ and consecutive radial order $n$, except if $\ell \geq 3$ modes are taken into account.

In order to test the hypothesis of modes of consecutive $n$ and the same degree $\ell$, the maximum separation of the observed frequencies $\left(f_{2}-f_{1}=0.94 \mathrm{mHz}\right)$ can be compared with the theoretical frequency distance between consecutive $n$ values of the same $\ell$. In Fig. 11 the frequency separation of consecutive order for $\ell=0,1,2,3$ is plotted as a function of time for the four sequences considered here.

The frequency distance between consecutive $n$ values for models of sequence A never reach $1 \mathrm{mHz}$ at any evolutionary state nor $\ell$ degree (Fig. 11). However, this frequency separation is considerably lower for other sequences. Slightly evolved models of sequence $B$, and sequence $\mathrm{C}$ at any evolutionary stage are able to explain $f_{2}-f_{1}$ as modes of the same degree and consecutive radial order. Sequence D seems to be less realistic, as only $\ell=2$ modes reach the observed separation. Since the theoretical frequency separation between $\ell=2$ and $\ell=1$ modes (see Fig. 9) is considerably greater than the small separations shown in Fig. 10, $f_{2}$ cannot be an $\ell=2$ mode in order to explain its close frequencies $f_{3}$ and $f_{4}$.

\section{Conclusions}

A new member of the rapid EC 14026 (or V361 Hya) pulsator class has been discovered. The observed electromagnetic 


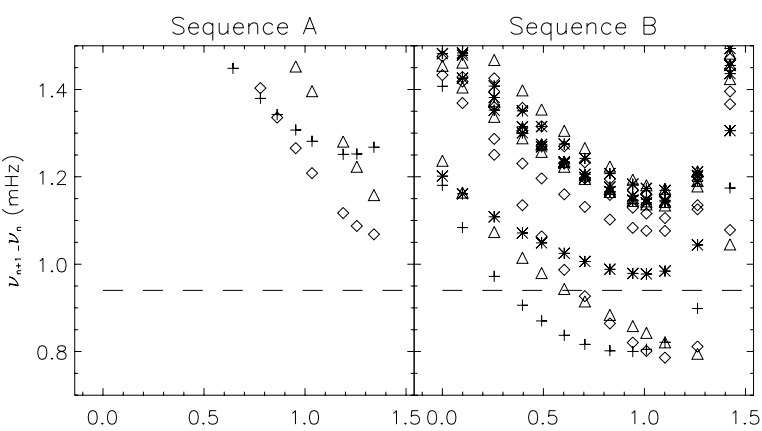

Sequence C

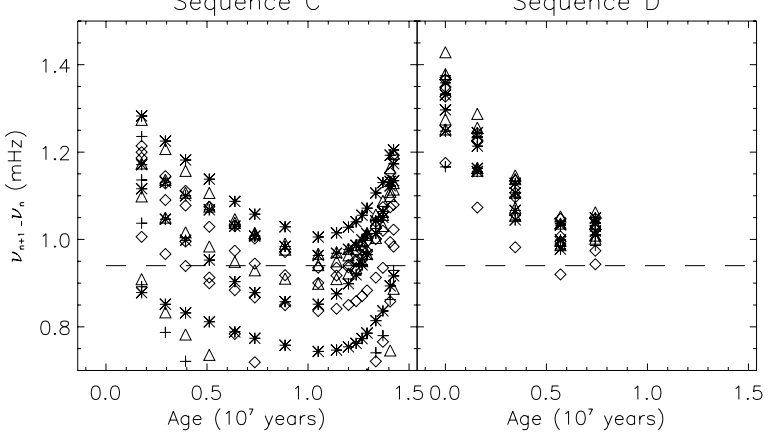

Fig. 11. Frequency separation between modes of consecutive radial order for $\ell=0$ (crosses), 1 (asterisks), 2 (rhombus) and 3 (triangles) as a function of time for the four sequences. The observed frequency separation $f_{2}-f_{1}=0.94 \mathrm{mHz}$ is overplotted as an horizontal broken line for comparison.

spectrum and the photometric magnitudes are well reproduced by a sdB+G5 system, with the companion star having $T_{\text {eff }}=$ $5500 \pm 500 \mathrm{~K}$ and the hot subdwarf $T_{\text {eff }}=32200 \pm 500 \mathrm{~K}$ and $\log g=5.73 \pm 0.10$ dex.

The frequency range of the detected pulsations seems to be in good agreement with those of other stars with similar temperature, for example PG $1219+534\left(T_{\mathrm{eff}}=33600 \mathrm{~K}\right.$, $\log g=5.8 \mathrm{dex}$ ), whose main detected modes lie in the range $6.7-7.8 \mathrm{mHz}$, or PG $1618+563 \mathrm{~B}\left(T_{\mathrm{eff}}=33900 \mathrm{~K}, \log g=\right.$ $5.8 \mathrm{dex}$ ), with two detected frequencies at 6.9 and $7.1 \mathrm{mHz}$. The number as well as the amplitude of the observed modes also seem to be consistent with other EC 14026 stars of similar parameters. Amplitude variations are detected, implying that either non resolved peaks are present or that real amplitude variations occur in PG $1657+416$. For any of these reasons, the possibility of finding additional excited modes in further observations cannot be discarded.

We compared observed and predicted frequencies using structural sdB models along different evolutionary sequences. The separation between the observed frequencies seems to be well reproduced by low order $(n=2-6)$ p-modes of structural models with physical parameters around those determined spectroscopically. Although we find that sequences produced by a single evolutionary channel with a total mass around $0.47 M_{\odot}$ are prefered to models with higher masses located in the same region of the $T_{\text {eff }}-\log g$ diagram, the limited number of models considered and frequencies detected are not sufficient to discount any of the proposed scenarios.

Acknowledgements. R.O. acknowledges Gobierno de Canarias for its financial support and Ángel R. López-Sánchez for his redden lights. A.U. acknowledges financial support from the Spanish MEC under research contract AYA2003-09499. This work has received financial support from the Spanish Plan Nacional del Espacio under project ESP2004-03855-C03-03. We thank Prof. Uli Heber for kindly providing the LTE spectral grids, and the anonymous referee for valuable suggestions that greatly improved the manuscript. The IAC 80 is operated on the island of Tenerife by the Instituto de Astrofísica de Canarias in the Spanish Observatorio del Teide. Based on observations made with the Nordic Optical Telescope, operated on the island of La Palma jointly by Denmark, Findland, Iceland, Norway and Sweden, in the Spanish Observatorio del Roque de los Muchachos of the Instituto de Astrofísica de Canarias.

\section{References}

Bergeron, P., Saffer, R. A., \& Liebert, J. 1992, ApJ, 394, 228

Brassard, P., Fontaine, G., Billères, M., et al. 2001, ApJ, 563, 1013

Charpinet, S., Fontaine, G., Brassard, P., \& Dorman, B. 1996, ApJ, 471, L103

Charpinet, S., Fontaine, G., Brassard, P., et al. 1997, ApJ, 483, L123

Charpinet, S., Fontaine, G., Brassard, P., et al. 2005a, A\&A, 443, 251

Charpinet, S., Fontaine, G., Brassard, P., Green, E. M., \& Chayer, P. 2005b, A\&A, 437, 575

Chayer, P., Fontaine, G., Fontaine, M., et al. 2004, in Extreme Horizontal Branch Stars and Related Objects, ed. P. Maxted, Ap\&SS, 291, 359

Claret, A. 1995, A\&AS, 109, 441

Green, R. F., Schmidt, F., \& Liebert, J. 1986, ApJS, 61, 305

Han, Z., Podsiadlowski, Ph., Maxted, P. F. L., Marsh, T. R., \& Ivanova, N. 2002, MNRAS, 336, 449

Han, Z., Podsiadlowski, Ph., Maxted, P. F. L., \& Marsh, T. R. 2003, MNRAS, 341,669

Harms, S. L., Reed, M., \& O'Toole, S. 2005, in Second Meeting on Hot Subdwarfs and Related Objects, ed. R. Østensen, Balt.A., 15, 252

Heber, U., Reid, I. N., \& Werner, K. 2000, A\&A, 363, 198

Heber, U., Moehler, S., Napiwotzki, R., Thejll, P., \& Green, M. 2002, 383, 938

Jiménez, R., \& MacDonald, J. 1996, MNRAS, 283, 271

Kilkenny, D., Koen, C., O’Donoghue, D., \& Stobie, R. S. 1997, MNRAS, 285, 640

Koen, C., Kilkenny, D., O’Donoghue, D., Van Wick, F., \& Stobie, R. S. 1997, MNRAS, 285, 645

Maxted, P. F. L., Marsh, T. R., Heber, U., et al. 2002, MNRAS, 333, 231

Munari, R., Sordo, R., Castelli, F., \& Zwitter, T. 2005, A\&A, 442, 1127

Oreiro, R., Pérez Hernández, F., Ulla, A., et al. 2005, A\&A, 438, 257

Østensen, R. 2000, Time Resolved CCD Photometry, Ph.D. Thesis, University of Troms $\emptyset$, Norway

Østensen, R. 2004, in Extreme Horizontal Branch Stars and Related Objects, ed. P. Maxted, Ap\&SS, 291, 263

Reed, M., Eggen, J. R., Zhou, A.-Y., et al. 2006, MNRAS, in press

Reimers, D. 1975, Mem. Soc. Roy. Sci. Liege, 8, 369

Saffer, R., Bergeron, P., Koester, D., \& Liebert, J. 1994, ApJ, 432, 351

Schlegel, D. J., Finkbeiner, D. P., \& Davis, M. 1998, ApJ, 500, 525

Silvotti, R., Janulis, R., Schuh, S. L., et al. 2002, A\&A, 389, 180

Stark, M., \& Wade, R. 2003, AJ, 126, 1455

Whitford, A. E. 1958, AJ, 63, 201 